\title{
AC 2008-2016: INCORPORATING HDL BASED DESIGN FLOW IN EET CURRICULUM
}

\section{MADDUMAGE KARUNARATNE, University of Pittsburgh - Johnstown}

Dr. Maddumage Karunaratne - Assistant Professor of Electrical Engineering Technology at the University of Pittsburgh at Johnstown. Dr. Karunaratne earned a Bachelor of Science degree from the University of Moratuwa, a Master of Science from the University of Mississippi, and a Ph.D. from the University of Arizona. He has extensive industry experience working in the Semiconductor industry in software development, Application Engineering, Design, Testing and Verification of IC components. His research and teaching interests include Semiconductor Testing and Verification, Low Power Design and Analysis, Digital and embedded systems, and Software engineering and IC Design automation.

\section{Gregory Dick, University of Pittsburgh -Johnstown}

Dr. Gregory M. Dick - Associate Professor and Head of Electrical Engineering Technology at the University of Pittsburgh at Johnstown. Dr. Dick holds degrees from the University of Pittsburgh, Stanford University, and the Pennsylvania State University, and is licensed in the Commonwealth of Pennsylvania. He has taught at Pitt-Johnstown for over 30 years. His areas of interest include Computing, Systems and Controls, Digital Signal Processing and the interface between technology and society. 


\title{
Incorporating HDL Based Design Flow in EET Curriculum
}

\begin{abstract}
:
Based on industry trends and advances in silicon manufacturing technology in recent years, it is becoming apparent that future electrical and computer engineers will most likely implement their digital designs using programmable logic devices such as CPLDs and FPGAs, rather than discreet IC components. They may also encounter electronic systems built on such devices in their engineering practice. Therefore, it has become necessary to incorporate related design techniques into courses even at the undergraduate level. This paper discusses the introduction of and teaching of such courses to undergraduates majoring in the Electrical Engineering Technology program at the University of Pittsburgh at Johnstown (UPJ).
\end{abstract}

The paper also describes several considerations taken into account during the adaptation of Hardware Description Languages (HDL) and automation based digital design flow to the UPJ curriculum. The demographics of UPJ's student population and their immediate careers suggest that most graduates do not pursue graduate studies in computer engineering, nor do they seek employment related to design and manufacture of integrated circuit components. As a result, a detailed in depth study of modern digital design methodologies is not a necessity.

The paper explains the course topics and the related laboratory assignments of the core digital electronics course for UPJ undergraduates. The results from a student survey taken at the end of the course to gauge the effectiveness of HDL and associated tools in learning digital electronics are also discussed. The paper elaborates on advantages and disadvantages of using HDL based circuit design in the undergraduate engineering technology curriculum as seen by students.

\section{Section I: Introduction:}

In the not too distant past, student assignments in digital design courses consisted primarily of paper designs, or at best such as in senior design projects they were a large morass of SSI and MSI (Small and Medium Scale Integration) silicon devices plugged on to circuit boards or wiring boards. More time was spent on debugging the connections and wirings than on actual design or in determining functionalities of the system. Even after HDLs such as VHDL have become standards and widely being used in industry, undergraduate academic curriculums in Electrical and Computer engineering were very slow to adopt them [1] because of the cost of the hardware and associated Computer Aided Design (CAD) tools required for digital designs.

Even those college level courses that utilized HDL, the designs were limited to use them only for design and simulation stages, and not for hardware implementations. This scenario started to change gradually with the advent of cheaper computers, inexpensive Programmable Logic Devices (PLDs), and the associated development environments. Although the availability and cost of using a programmable logic design methodology was initially prohibitive for most academic environments, this is no longer the case. The 
flexibility of PLDs and the availability of inexpensive CAD tools with huge academic discounts from the industry that support these devices make them ideal for a wide range of digital design courses. Developing designs in hardware languages and associated methodologies teach students the skills to implement large and complex circuits using high level abstraction and adds marketable tools to the student's skill set.

Prior to adaptation of HDL based flows, digital designs were described on paper or on graphical schematics CAD tools by connecting the vendor supplied icons of building blocks named cells. It was of such low level and so tedious that the productivity was very low. Compared to schematic-based design, HDLs provide designers with the following advantages [3]:

- Designs can be transcribed at various levels of abstraction. Designers can write their register transfer language (RTL) descriptions without choosing a specific fabrication technology. Logic synthesis tools can automatically convert the design to any fabrication technology.

- Describing designs in HDLs allows functional verification of the design early in the design cycle. Because designers work at the RTL level, they can optimize and modify the RTL description until it meets the desired functionality. Most design bugs are eliminated at this point. This significantly decreases design cycle time.

- Designing with HDLs is analogous to computer programming. A textual description with comments is an easier way to develop and debug circuits. Gatelevel schematics can be very cumbersome and functionally opaque for complex digital designs.

Based on industry trends it is becoming apparent that future electrical and computer technology engineers will most likely implement their digital designs using programmable logic devices such as CPLDs and FPGAs, rather than discreet IC components, while only a very minute fraction of digital designs would be made in either Application Specific Integrated Circuit (ASIC) technology or in discrete logic components such as gates and flipflops. From the papers published in recent years in various venues [4 8] it is apparent that the academic departments are adding new courses or content to teach HDL based design flows at some level to undergraduate EET students.

Therefore, to help the graduates to be competent and competitive in their major disciplines, UPJ EET majors are exposed to the HDL based digital design techniques at the undergraduate level. There were several key considerations taken into account during the adaptation of Hardware Description Languages (HDL) and automation based digital design flow to the UPJ curriculum. The demographics of UPJ's student population and their immediate careers suggest that most graduates do not pursue graduate studies in computer engineering, nor do they seek employment related to design and manufacture of Integrated Circuit components.

The primary objective of EET program at UPJ has been to provide a broad skill sets to graduates to work in various local and regional industries including the defense sector, and therefore the EET curriculum does not provide formally declared specialization areas. The 
core curriculum schedule accommodates only one digital design course namely EET 1161 "Digital Electronics" in the junior year. In the senior year an elective is offered that looks deeper in to system level design using software/hardware co design, design verification, testing, design for test, and advanced design topics. The EET 1161 course is accompanied by its associated laboratory course, EET 1061. The academic calendar of UPJ provides 15 weeks for the courses and about three hours contact time a week for each course.

Section II of the paper describes the Course objectives and Content along the description of selected sections of the typical HDL based design flow to suit the UPJ EET curriculum; Section III elaborates on laboratory experiments and project assignments while Section IV ponders upon a survey done on students in the course at the end of the semester. Section V states concluding remarks with the future treads.

\section{Section II : Course objectives and Content}

The objective of the course and the lab is to provide students with a skill set so they would be able to analyze, develop, design and implement a reasonable large and complex digital logic circuit using either discrete components or a PLD. Table 1 shows the course topics and the relevant lab topics for the course.

The theme of the course may be viewed as consisting of the following four sections:

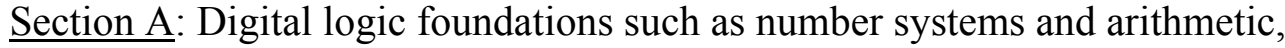
Boolean algebra and expressions;

Section B: Combinational logic design;

Section C: Sequential logic design;

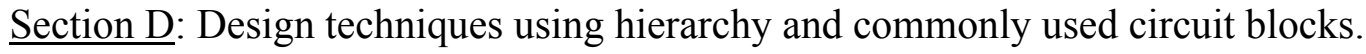

The course topics are not grouped in to those four sections; rather the course is organized into topics in the sequential order as shown in TABLE 1 to provide a progressive learning flow.

\begin{tabular}{|c|l|l|}
\hline 1 & Lecture Topics & Lab Topics \\
\hline 1 & $\begin{array}{l}\text { Information representation in Digital } \\
\text { Systems: decimal, binary, and hexadecimal } \\
\text { systems; }\end{array}$ & $\begin{array}{l}\text { Lab 1: introduction of logic } \\
\text { families, digital trainers, threshold } \\
\text { voltages, etc. }\end{array}$ \\
\hline 2 & $\begin{array}{l}\text { Boolean Algebra and function minimizations } \\
\text { using Karnaugh Maps, etc }\end{array}$ & $\begin{array}{l}\text { Lab 2 3: Boolean functions and } \\
\text { Boolean algebraic manipulations, } \\
\text { minimizations }\end{array}$ \\
\hline 3 & $\begin{array}{l}\text { Combinational Logic elements: Basic and } \\
\text { complex logic gates; high impedance output } \\
\text { gates; partitioning for single and multi level } \\
\text { optimizations. }\end{array}$ & $\begin{array}{l}\text { Lab 4 5: Combinational logic } \\
\text { design, design partitioning, } \\
\text { Boolean manipulations }\end{array}$ \\
\hline 4 & $\begin{array}{l}\text { Combinational Logic Design and Functions: } \\
\begin{array}{l}\text { Top-down and bottom-up design and } \\
\text { hierarchy; Design automation and }\end{array}\end{array}$ & $\begin{array}{l}\text { Lab 6: Combinational logic } \\
\text { design with gate level Verilog } \\
\text { HDL, testbench creation using }\end{array}$ \\
\hline
\end{tabular}




\begin{tabular}{|c|c|c|}
\hline & $\begin{array}{l}\text { implementation methods; Role of Hardware } \\
\text { Description Languages in design } \\
\text { automation; Technology libraries; } \\
\text { propagation delays; simulation and } \\
\text { verification of circuits; decoders; } \\
\text { multiplexors; encoders; Logic circuit } \\
\text { synthesis using pre-designed function } \\
\text { blocks. }\end{array}$ & $\begin{array}{l}\text { Verilog procedural blocks, } \\
\text { Verilog models of standard } \\
\text { hardware components to emulate } \\
\text { hardware for functionally } \\
\text { verifying the designed circuit. } \\
\text { Lab 7: Parameterized designs, } \\
\text { hierarchical designs, Verilog } \\
\text { testbench developments, and a } \\
\text { case for non-exhaustive testing }\end{array}$ \\
\hline 5 & $\begin{array}{l}\text { Arithmetic Circuits: Iterative combinational } \\
\text { circuits; adders; subtracters; arithmetic } \\
\text { overflow; multiplication by constants; } \\
\text { increments and decrements. }\end{array}$ & $\begin{array}{l}\text { Lab 8: Sequential adder using } \\
\text { iterative adder blocks implemented } \\
\text { in hardware LSI/MSI silicon parts. }\end{array}$ \\
\hline 6 & $\begin{array}{l}\text { Sequential Circuit Design: sequential logic } \\
\text { elements and timing diagrams; sequential } \\
\text { circuit analysis and design; state diagrams; } \\
\text { state assignments; one-hot encoding; } \\
\text { Different abstract levels of Verilog HDL } \\
\text { language, simulation and verification of } \\
\text { sequential designs. }\end{array}$ & $\begin{array}{l}\text { Lab 9: Design of a finite state } \\
\text { machine in HDL and verification by } \\
\text { simulation with a testbench. } \\
\text { Lab 10: Implementation of an } \\
\text { already verified sequential circuit on } \\
\text { a programmable logic device, such } \\
\text { as Xilinx CPLD. }\end{array}$ \\
\hline 7 & $\begin{array}{l}\text { Sequential Design Blocks: Analysis and } \\
\text { design of data registers, universal shift } \\
\text { registers, ripple and synchronous binary } \\
\text { counters; Sequential design partitions as data } \\
\text { and control sections. }\end{array}$ & $\begin{array}{l}\text { Project: Design of a sequential } \\
\text { circuit in hierarchical partitions } \\
\text { based on control and data sections; } \\
\text { demonstration of its } \\
\text { implementation on CPLD. }\end{array}$ \\
\hline
\end{tabular}

Table 1: Digital Design Course Outline

Students learn the materials in Section A in the first few classes of the course so that they know the algebra of the foundation of digital designs rather than merely relying on CAD tools for logic optimization. After basic Boolean algebra students learn basic logic elements and Verilog HDL is first introduced at that time to describe combinational primitive logic gates. Students who take the course have already done C-programming and the similarity between Verilog HDL and C language helps significantly as does the prior exposure to binary and hexadecimal number systems.

The inventors of Verilog language [11] wanted a language with syntax similar to the C programming language so that it would be familiar to engineers and readily accepted. The language is case-sensitive, has a preprocessor like $\mathrm{C}$, and the major control flow keywords, such as "if" and "while", are similar. The formatting mechanism in the printing routines and language operators and their precedence are also similar. Verilog HDL was selected as the language over VHDL primarily because all the students will have gained the prerequisite experience in $\mathrm{C}$ programming for one semester.

After the theory and techniques in Combinational Logic Design (Section B) and Functions have been covered, Verilog is introduced more formally with the concepts of 
abstraction and encapsulation (of modules). As Table 1 outlines, students learn hierarchical design and design reuse; automation of the design flow for ASIC designs using technology libraries; delay modeling at gate level, role of Hardware Description Languages in design automation; Technology libraries; simulation and verification of circuits; decoders; multiplexors; encoders; and other typical functional blocks. Verilog HDL is introduced via examples for gate level designs already completed in class. In the Verilog HDL itself, students learn gate level and RTL level circuit modeling, concurrent assignments for combinational designs, and procedural blocks for testbenches.

More semantics complexities arise in Verilog when Sequential Circuits (Section C) are described at RTL level which is the preferred level of abstraction for sequential designs when targeting Complex PLDs (CPLDs). Students learn classical sequential circuit design and then use Verilog to describe them rather than directly coding from the problem specifications. However they are introduced to the RTL coding style of finite state machines which follows the switch-case statements in C language. For Section-d, the sequential circuit blocks such as counters, shift registers, and memory modules are taught along with Verilog coding styles for them at different abstract levels.

Although Verilog HDL offers an extensive feature set for modeling digital circuits at any abstract level, due to time constraints, students do not get the opportunity to study switch level modeling and signal strengths (except the high impedance state); behavior level modeling; delay modeling although testbenches use some delays in between driving value sets; and signal timing constraints in technology library cells; or mapping of the circuit to library cells for ASICs design flow.

\section{Section III: Laboratory Experiments and Class Projects.}

Lab assignments reflect on material covered in the theory class. Students use ModelSim [9] for simulation and validation of their design work. They develop testbenches for verification of the designs including self checking testbenches where outputs and expected values are compared by simulation itself indicating any errors and pass/fail status of the entire test. As table 1 shows, lab assignment 7 introduces parameterized designs to allow resizing of the same module to build larger circuits. It also demonstrates a case for non-exhaustive testing of a seemingly simple circuit.

Toward the end of the course, the students synthesize a circuit that they already designed and verified in a prior lab, into a Xilinx CPLD using ISE CAD software [13]. The final project is to partition a design from a verbal specification into data and control sections to be designed as individual modules with hierarchy.

In this course, the Verilog language is treated as a tool, and the effort and emphasis placed on teaching Verilog can be estimated to be about $20 \%$ which can not be increased. This, because it is the only digital design core course UPJ EET students take and any increase in Verilog content would adversely affect the teaching of design principles. Also adding another core course simply to expand the amount of Verilog language covered in the curriculum is not needed because such a broad spectrum is required only for those who would be working in design for manufacture of integrated circuit components, and not for system designers or system integrators of logic devices. 
The demographics of UPJ's student population and the companies they typically work for after graduation suggest that most UPJ graduates do not pursue graduate studies in computer engineering or electrical engineering. Also they do not seek employment in niche areas related to design, manufacture, or testing of integrated circuit components such as microprocessors, video chips, etc. On the other hand those who have gone to graduate studies do not find lack of the depth in certain areas adversely affect pursuance of their desired area of study in graduate programs. As a result, a much broader and deeper study of modern digital design methodologies is not a necessity for most UPJ EET undergraduate students.

Since the teaching of Verilog HDL is not the major emphasis nor it is taught heavily, students do not show lack of interest or boredom in the course. Although both students and the instructor like to increase the amount of time in learning Verilog, the time limits and core learning required prevents it. Both parties however think that students are exposed to a sufficient amount of Verilog HDL in design abstraction levels, coding styles, and implementation options that students would be able to self-learn and become effective in implementing large and complex designs on programmable devices or other means.

\section{Section IV: Student's feedback}

Students were surveyed at the end of the course to gauge the effectiveness of HDL and associated tools in learning digital electronics, although the real benefit would occur when they graduate and find employment in computer related fields. The familiarity and exposure to a HDL based design methods and programmable logic devices would enable them for life-long learning in changing technologies.

The survey was conducted on a small class (UPJ typically have classes of less than 20 students) at the end of the EET1161 lecture course and EET1061 labs, and all of the students participated. The results are summarized qualitatively in Table 2 . The responses were given based on the typical rubrics of 1 to 5 (1-strongly disagree, 2-disagree, 3-neutral, 4-agree, 5strongly disagree). Column 2 of Table 2 lists what each question was trying to learn from the students. They were not asked in a direct way since students would probably select rubric values just to appease the instructor. $3^{\text {rd }}$ column is the average value of the rubric values circled by each student. Final column in Table 2 attempts to give a qualitative meaning to the data in column 3 so that whether the Verilog HDL content its delivery methods need to be changed for better learning of HDL based design flows.

Students clearly say that HDL removes or reduces the visualization of the circuit which is inherent in a text based description. However, there are CAD tools that could process the textual description of the circuit and display a schematic or a block diagram of the circuit. One such tool is Xilinx ISE software used in configuring CPLDs that students already use. It may be desired to instruct future students of the course to generate schematics for their HDL circuit descriptions.

Among the other questions posed to the students in Table 2, for items 2 through 6 they were neutral or close to being neutral, and therefore they received the qualitative value of "ok" implying no action is really required at this point. The question 7 in Table 2 carries the 
qualitative measure of "yes" which states that future students would benefit by increased Verilog HDL content in the course, at least that is the message from the last semester EET students in EET1161/1061 course. Answers to the questions 8 and 9 show that students are comfortable using the HDL language because otherwise they would have more strongly indicated the desire to work in a group setting for lab assignments. Looking at the last question, it is an encouragement to see students showing a desire to learn new technologies which in turn encourages addition of more Verilog HDL content in the curriculum. The obvious disadvantage of introducing HDL based flow would be that it takes reduces the time allowed to teach certain topics in depth and prevents some more being added. With the Cprogramming background students have, a major disadvantage of having to learn programming techniques anew was not present in this case.

\begin{tabular}{|l|l|l|l|}
\hline & Essence of the question asked & Average & Qualitative \\
\hline 1 & Student likes to draw circuits (schematics) & 4 & yes \\
\hline 2 & Student likes to use Verilog to describe circuits & 3.2 & ok \\
\hline 3 & Student feels the syntax of Verilog too rigid & 3.5 & ok \\
\hline 4 & $\begin{array}{l}\text { Student would like a much relaxed grammar for Verilog } \\
\text { HDL }\end{array}$ & 3.3 & ok \\
\hline 5 & $\begin{array}{l}\text { Encapsulation in Verilog forced me to think of complete } \\
\text { circuit (ports, wires, etc) }\end{array}$ & 3.5 & ok \\
\hline 6 & $\begin{array}{l}\text { Student } \text { missed feeling of accomplishment by not seeing } \\
\text { the circuits (lights) }\end{array}$ & 3.5 & ok \\
\hline 7 & $\begin{array}{l}\text { Student would have benefited more in the course, if } \\
\text { student knew more Verilog }\end{array}$ & 4 & yes \\
\hline 8 & $\begin{array}{l}\text { Student has } \text { own personal computer with a copy of } \\
\text { ModelSim (free simulator) }\end{array}$ & 4.8 & free \\
\hline 9 & Student likes to work alone on lab assignments & 2.7 & ok \\
\hline 10 & Student is not keen on learning new techniques in class & 2 & yes \\
\hline
\end{tabular}

Table 2: Student Survey Results

\section{Section V: Conclusion}

With the current complexity levels of functionality in digital circuits and the abundance of inexpensive programmable logic devices with a wide range of circuit characteristics, HDL based design techniques are rapidly replacing the traditional circuits built using discrete integrated circuit parts. Using HDLs designs can be described at any abstract level, and designers can write their circuit descriptions without choosing either a specific fabrication technology in the case of Application Specific ICs or a particular programmable logic device. CAD tools can easily convert designs to future technologies; functional verification of the design can be done very early in the design cycle; and a significant time reduction in the design cycle can be accomplished by using HDLs [10].

It is evident that the time has arrived to introduce HDL training into the undergraduate EET curricula of UPJ. The incorporation of Verilog HDL based design implementation techniques to UPJ students helps their learning of the course material, and also makes the UPJ graduates more marketable. By providing electrical engineering technology graduates skills associated with advanced and efficient design methods, they will be trained to see the 
need to implement and promote technological changes at their work place throughout their careers.

\section{References:}

[1] VHDL International, "VHDL International University Usage Survey,” VHDL International, Santa Clara, CA, 1995.

[2] S. Palnitlear, Verilog HDL. Englewood Cliffs, NJ: Prentice-Hall, 1996.

[3] Fundamentals of Digital Logic with Verilog Design By Stephen Brown, Zvonko Vranesic, Published 2002 McGraw-Hill Professional

[4] S. M. Sait, "Integrating UAHPL-DA systems with VLSI design tools to support VLSI DA courses," IEEE Trans. Educ., vol. 35, pp. 321-329, Nov. 1992.

[5] Norihiro Fujii, “Top-Down eLearning Tools for Hardware Logic Design," Proceedings of the 2003 International Conference on Cyberworlds, 2003 IEEE

[6] Oliver, J.P., Haim, F., Fernandez, S., Rodriquea, J., Rolando, P., "Hardware Lab at Home Possible with Ultra Low Cost Boards", Proceedings: International Conference on Microelectronic Systems Education, June 2005, pp. 19-20.

[7] Nestor, J.A., "Teaching Computer Organization with HDLs: An Incremental Approach", Proceedings: International Conference on Microelectronic Systems Education, June 2005, pp. 77-78.

[8] Bryan J. Mealy, "Work In Progress: Computer Design for Intermediate-Level Digital Systems Course," 36th ASEE/IEEE Frontiers in Education Conference, 2006

[9] ModelSim reference at http://www.xilinx.com/ise/optional_prod/mxe.htm

[10] D. Gajski, Silicon Compilation. Reading, MA: Addison-Wesley, 1988.

[11] IEEE standard 1364, "IEEE Standard Hardware Description Language Based on Verilog", IEEE Computer Society, 1996.

[12] QuickHDL User's and Reference Manual, Mentor Graphics, 1996.

[13] Xilinx website: www.xilinx.com

[14] Digilent Inc. website: www.digilentinc.com

[15] Thomas \& Moorby, “The Verilog Hardware Descroption Language," Kluwer Academic Press, 1996. 